Acta Crystallographica Section E

Structure Reports

Online

ISSN 1600-5368

\section{2,2-Dimethyl-5-triphenylmethyl-1,3- dioxane}

\section{Min Zhang, ${ }^{*}$ Xian-You Yuan and Xing-Ming Liu}

Department of Biology and Chemistry, Hunan University of Science and Engineering, Yongzhou Hunan 425100, People's Republic of China

Correspondence e-mail: zm_zmcm@sina.com

Received 30 November 2008; accepted 5 January 2009

Key indicators: single-crystal X-ray study; $T=298 \mathrm{~K}$; mean $\sigma(\mathrm{C}-\mathrm{C})=0.003 \AA$; $R$ factor $=0.055 ; w R$ factor $=0.193 ;$ data-to-parameter ratio $=17.4$.

The title compound, $\mathrm{C}_{25} \mathrm{H}_{26} \mathrm{O}_{2}$, crystallizes with two crystallographically independent molecules in the asymmetric unit. The differences between the two molecules are marginal. The three benzene rings of each molecule are in a propeller orientation and the 1,3-dioxane ring adopts a chair conformation.

\section{Related literature}

For the synthesis of the compound, see: Whilt \& Finnerty (1961); Yuan et al. (2007); Wang et al. (1995). For applications of this class of compounds, see: Wang, Yuan, Liu et al. (1996); Wang, Yuan, Lei \& Liu (1996); Yuan et al. (2005). For related crystal structures, see: Chuprunov et al. (1981); Yuan et al. (2008).

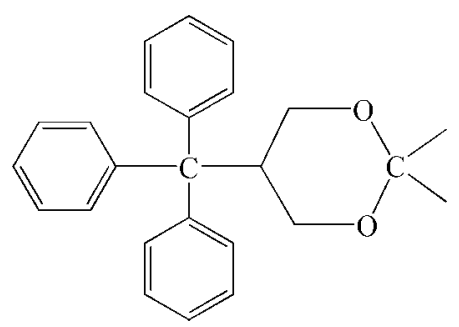

\section{Experimental}

Crystal data

$\mathrm{C}_{25} \mathrm{H}_{26} \mathrm{O}_{2} \quad M_{r}=358.47$
Triclinic, $P \overline{1}$

$a=10.7252(18) \AA$

$b=11.6933(19) \AA$

$c=15.840(3) \AA$

$\alpha=89.574(3)^{\circ}$

$\beta=88.906(3)^{\circ}$

$\gamma=86.427(3)^{\circ}$

$V=1982.3(6) \AA^{3}$

$Z=4$

Mo $K \alpha$ radiation

$\mu=0.07 \mathrm{~mm}^{-1}$

$T=298(2) \mathrm{K}$

$0.45 \times 0.38 \times 0.29 \mathrm{~mm}$

\section{Data collection}

Bruker SMART CCD area-detector diffractometer

Absorption correction: multi-scan (SADABS; Sheldrick, 1996)

$$
T_{\min }=0.967, T_{\max }=0.979
$$

\section{Refinement}

$R\left[F^{2}>2 \sigma\left(F^{2}\right)\right]=0.055$

$w R\left(F^{2}\right)=0.193$

$S=1.07$

8543 reflections

16710 measured reflections 8543 independent reflections 5142 reflections with $I>2 \sigma(I)$ $R_{\text {int }}=0.028$

Data collection: SMART (Bruker, 1997); cell refinement: SAINT (Bruker, 1997); data reduction: $S A I N T$; $\operatorname{program}(\mathrm{s})$ used to solve structure: SHELXS97 (Sheldrick, 2008); program(s) used to refine structure: SHELXL97 (Sheldrick, 2008); molecular graphics: SHELXTL (Sheldrick, 2008); software used to prepare material for publication: SHELXTL.

This work was financially supported by the Key Scientific Research Project of the Hunan Provincial Education Department (No. 08 A023) and the Key Subject Construction Project of Hunan Province (No. 2006-180).

Supplementary data and figures for this paper are available from the IUCr electronic archives (Reference: BT2825).

\section{References}

Bruker (1997). SMART and SAINT. Bruker AXS Inc., Madison, Wisconsin, USA.

Chuprunov, E. V., Tarkhova, T. N., Korallova, T. Y., Simonov, M. A. \& Belov, W. V. (1981). Zh. Strukt. Khim. 22, 191-192.

Sheldrick, G. M. (1996). SADABS. University of Göttingen, Germany.

Sheldrick, G. M. (2008). Acta Cryst. A64, 112-122.

Wang, G. W., Yuan, X. Y., Lei, X. G. \& Liu, Y. C. (1996). Chin. J. Appl. Chem. 11, 114-115.

Wang, G. W., Yuan, X. Y., Liu, Y. C., Guo, Q. X. \& Lei, X. G. (1996). Indian J. Chem. Sect. B, 35, 583-585.

Wang, G. W., Yuan, X. Y., Liu, Y. C., Lei, X. G. \& Guo, Q. X. (1995). J. Am. Oil Chem. Soc. 72, 83-87.

Whilt, J. W. \& Finnerty, J. L. (1961). J. Org. Chem. 26, 2173-2177.

Yuan, X. Y., Yang, N. F., Luo, H. A. \& Liu, Y. J. (2005). Chin. J. Org. Chem. 25, 1049-1052.

Yuan, X.-Y., Zhang, M. \& Ng, S. W. (2008). Acta Cryst. E64, o1314.

Yuan, X. Y., Zhang, M. \& Yuan, L. (2007). Chin. J. Org. Chem. 27, 1600-1604. 


\section{supporting information}

Acta Cryst. (2009). E65, o304 [doi:10.1107/S1600536809000294]

\section{2,2-Dimethyl-5-triphenylmethyl-1,3-dioxane}

\section{Min Zhang, Xian-You Yuan and Xing-Ming Liu}

\section{S1. Comment}

The title compound was synthesized to be use as a intermediate in surface activating reagent syntheses. The compound belongs to a class of 1,3-dioxane derivatives that have application in fine chemical medicine such as biology pharmacy (Wang, Yuan, Liu et al., 1996) and cosmetic industry (Wang, Yuan, Lei \& Liu, 1996; Yuan et al., 2005).

The title compound (Fig. 1) crystallizes with two crystallographically independent molecules per asymmetric unit. Differences between the two molecules are marginal. The three benzene rings of each molecule are in a propeller orientation and the 1,3-dioxane ring adopts a chair conformation. The structure is similar to that reported (Chuprunov et al., 1981; Yuan et al., 2008).

\section{S2. Experimental}

A mixture of $0.24 \mathrm{~g}$ (5.0 mmol) of 2,2-bis(hydroxymethyl)-1,3-propanediol, $0.10 \mathrm{~g}$ of phosphotungstic acid supported on actived carbon and $10 \mathrm{ml}$ of acetone were added to a round bottom flask of $50 \mathrm{ml}$ and heated under microwave irradiation condition for $3 \mathrm{~min}$, then $5 \mathrm{ml}$ benzene was added to this mixture. Then, this solution was heated, filtrated, and washed by using hot benzene. After benzene was evaporated and the sample was cooled. The resultant solid was recrystallized from anhydrous ethanol to give $1.1 \mathrm{~g}(58 \%)$ of white solid.

\section{S3. Refinement}

$\mathrm{H}$ atoms were placed in calculated positions ( $\mathrm{C}-\mathrm{H} 0.93$ to $0.98 \AA$ ) and were included in the refinement in the riding model approximation, with $U_{\text {iso }}(\mathrm{H})$ set to $1.2 U_{\text {eq }}(\mathrm{C})$ or $1.5 U_{\text {eq }}\left(\mathrm{C}_{\text {methyl }}\right)$. 

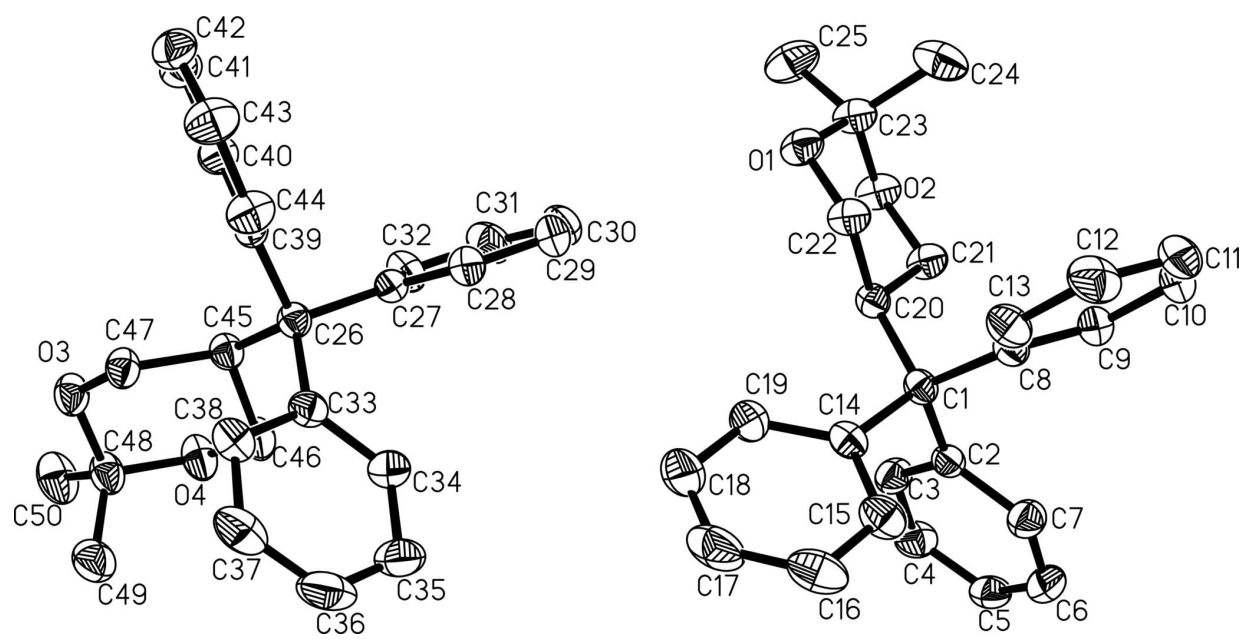

Figure 1

The molecular structure of the title compound, showing displacement ellipsoids at the $30 \%$ probability level. H-atoms have been excluded for clarity.

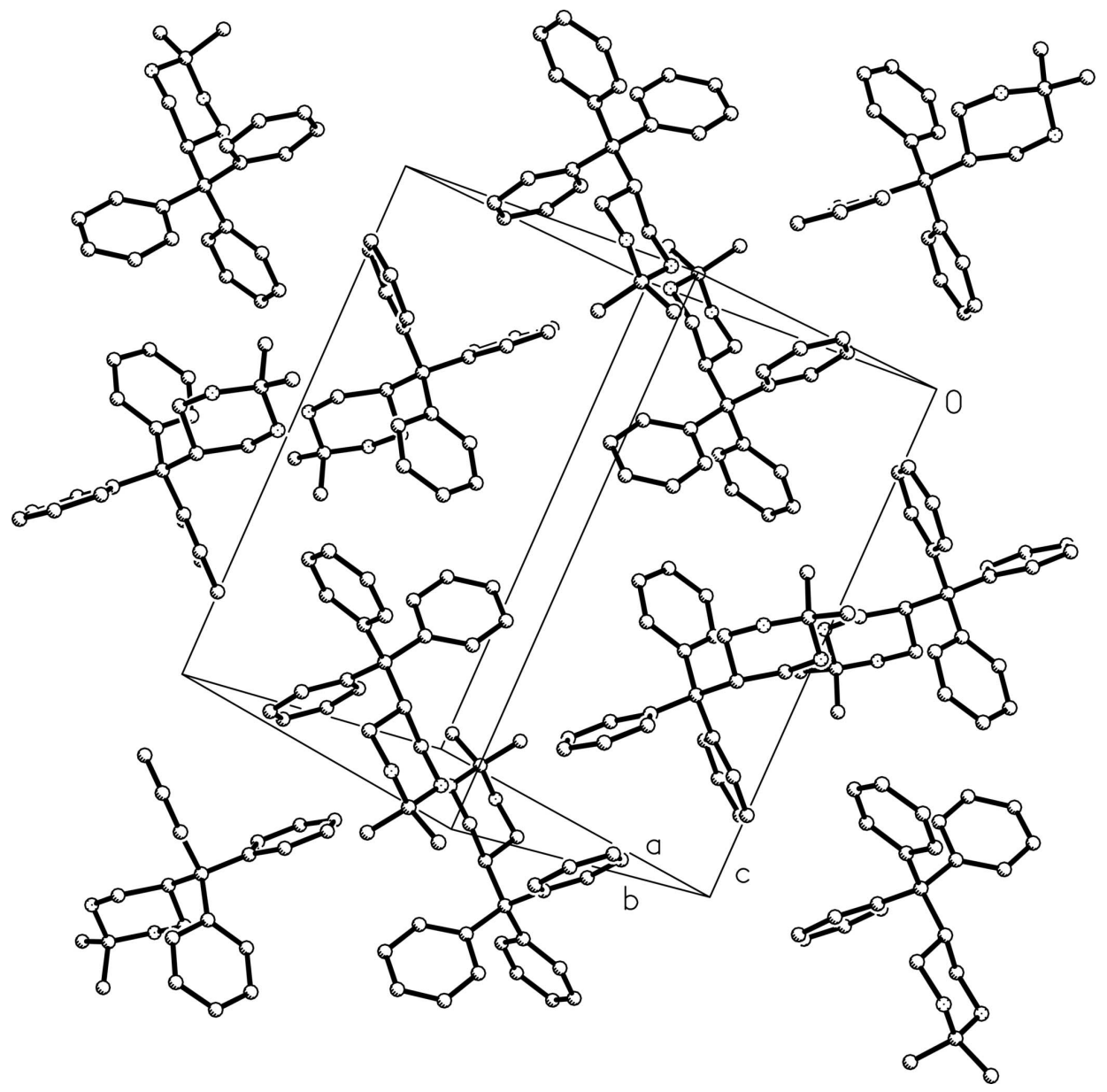

Figure 2

A view of the packing of the title compound. 


\section{2,2-Dimethyl-5-triphenylmethyl-1,3-dioxane}

Crystal data

$$
\begin{aligned}
& \mathrm{C}_{25} \mathrm{H}_{26} \mathrm{O}_{2} \\
& M_{r}=358.47 \\
& \text { Triclinic, } P \overline{1} \\
& a=10.7252(18) \AA \\
& b=11.6933(19) \AA \\
& c=15.840(3) \AA \\
& \alpha=89.574(3)^{\circ} \\
& \beta=88.906(3)^{\circ} \\
& \gamma=86.427(3)^{\circ} \\
& V=1982.3(6) \AA^{3}
\end{aligned}
$$

\section{Data collection}

Bruker SMART CCD area-detector diffractometer

Radiation source: fine-focus sealed tube Graphite monochromator $\varphi$ and $\omega$ scans

Absorption correction: multi-scan (SADABS; Sheldrick, 1996)

$T_{\min }=0.967, T_{\max }=0.979$

\section{Refinement}

Refinement on $F^{2}$

Least-squares matrix: full

$R\left[F^{2}>2 \sigma\left(F^{2}\right)\right]=0.055$

$w R\left(F^{2}\right)=0.193$

$S=1.07$

8543 reflections

491 parameters

0 restraints

Primary atom site location: structure-invariant direct methods
$Z=4$

$F(000)=768$

$D_{\mathrm{x}}=1.201 \mathrm{Mg} \mathrm{m}^{-3}$

Mo $K \alpha$ radiation, $\lambda=0.71073 \AA$

Cell parameters from 5597 reflections

$\theta=2.2-27.0^{\circ}$

$\mu=0.07 \mathrm{~mm}^{-1}$

$T=298 \mathrm{~K}$

Prism, colorless

$0.45 \times 0.38 \times 0.29 \mathrm{~mm}$

16710 measured reflections

8543 independent reflections

5142 reflections with $I>2 \sigma(I)$

$R_{\text {int }}=0.028$

$\theta_{\text {max }}=27.2^{\circ}, \theta_{\min }=1.3^{\circ}$

$h=-13 \rightarrow 13$

$k=-14 \rightarrow 14$

$l=-20 \rightarrow 20$

Secondary atom site location: difference Fourier map

Hydrogen site location: inferred from neighbouring sites

$\mathrm{H}$-atom parameters constrained

$w=1 /\left[\sigma^{2}\left(F_{\mathrm{o}}^{2}\right)+(0.1105 P)^{2}\right]$

where $P=\left(F_{\mathrm{o}}^{2}+2 F_{\mathrm{c}}^{2}\right) / 3$

$(\Delta / \sigma)_{\max }<0.001$

$\Delta \rho_{\max }=0.20$ e $\AA^{-3}$

$\Delta \rho_{\min }=-0.24$ e $\AA^{-3}$

\section{Special details}

Geometry. All e.s.d.'s (except the e.s.d. in the dihedral angle between two 1.s. planes) are estimated using the full covariance matrix. The cell e.s.d.'s are taken into account individually in the estimation of e.s.d.'s in distances, angles and torsion angles; correlations between e.s.d.'s in cell parameters are only used when they are defined by crystal symmetry. An approximate (isotropic) treatment of cell e.s.d.'s is used for estimating e.s.d.'s involving 1.s. planes.

Refinement. Refinement of $F^{2}$ against ALL reflections. The weighted $R$-factor $w R$ and goodness of fit $S$ are based on $F^{2}$, conventional $R$-factors $R$ are based on $F$, with $F$ set to zero for negative $F^{2}$. The threshold expression of $F^{2}>\sigma\left(F^{2}\right)$ is used only for calculating $R$-factors(gt) etc. and is not relevant to the choice of reflections for refinement. $R$-factors based on $F^{2}$ are statistically about twice as large as those based on $F$, and $R$ - factors based on ALL data will be even larger.

Fractional atomic coordinates and isotropic or equivalent isotropic displacement parameters $\left(\AA^{2}\right)$

\begin{tabular}{lllll}
\hline & $x$ & $y$ & $z$ & $U_{\text {iso }} * U_{\text {eq }}$ \\
\hline O1 & $0.35990(12)$ & $0.60731(12)$ & $0.01269(8)$ & $0.0549(4)$ \\
O2 & $0.14384(12)$ & $0.63492(12)$ & $0.01747(9)$ & $0.0563(4)$ \\
C1 & $0.23664(16)$ & $0.38835(15)$ & $0.17018(10)$ & $0.0397(4)$ \\
C20 & $0.24344(15)$ & $0.49994(15)$ & $0.11561(10)$ & $0.0409(4)$
\end{tabular}




\begin{tabular}{|c|c|c|c|c|}
\hline $\mathrm{H} 20$ & 0.2502 & 0.5634 & 0.1550 & $0.049 *$ \\
\hline $\mathrm{C} 2$ & $0.11404(16)$ & $0.39031(15)$ & $0.22318(10)$ & $0.0411(4)$ \\
\hline $\mathrm{C} 14$ & $0.34210(17)$ & $0.37887(16)$ & $0.23626(11)$ & $0.0447(4)$ \\
\hline $\mathrm{C} 8$ & $0.24895(18)$ & $0.28587(15)$ & $0.10870(11)$ & $0.0445(4)$ \\
\hline $\mathrm{C} 22$ & $0.35745(17)$ & $0.49994(17)$ & $0.05630(12)$ & $0.0494(5)$ \\
\hline $\mathrm{H} 22 \mathrm{~A}$ & 0.4330 & 0.4868 & 0.0886 & $0.059 *$ \\
\hline $\mathrm{H} 22 \mathrm{~B}$ & 0.3543 & 0.4385 & 0.0158 & $0.059^{*}$ \\
\hline C9 & $0.1479(2)$ & $0.25685(17)$ & $0.06129(12)$ & $0.0541(5)$ \\
\hline H9 & 0.0701 & 0.2948 & 0.0705 & $0.065^{*}$ \\
\hline $\mathrm{C} 21$ & $0.12987(17)$ & $0.53031(17)$ & $0.06175(12)$ & $0.0510(5)$ \\
\hline $\mathrm{H} 21 \mathrm{~A}$ & 0.1196 & 0.4694 & 0.0217 & $0.061 *$ \\
\hline H21B & 0.0555 & 0.5374 & 0.0976 & $0.061^{*}$ \\
\hline $\mathrm{C} 5$ & $-0.1020(2)$ & $0.39266(19)$ & $0.32589(12)$ & $0.0564(5)$ \\
\hline H5 & -0.1744 & 0.3936 & 0.3592 & $0.068^{*}$ \\
\hline $\mathrm{C} 3$ & $0.06172(19)$ & $0.49044(17)$ & $0.25835(12)$ & $0.0532(5)$ \\
\hline $\mathrm{H} 3$ & 0.0995 & 0.5588 & 0.2481 & $0.064 *$ \\
\hline $\mathrm{C} 13$ & $0.3626(2)$ & $0.22658(18)$ & $0.09322(12)$ & $0.0576(5)$ \\
\hline H13 & 0.4319 & 0.2440 & 0.1240 & $0.069 *$ \\
\hline $\mathrm{C} 15$ & $0.3606(2)$ & 0.27827 (19) & $0.28297(13)$ & $0.0598(5)$ \\
\hline H15 & 0.3095 & 0.2182 & 0.2740 & $0.072 *$ \\
\hline $\mathrm{C} 7$ & $0.0561(2)$ & $0.29087(17)$ & $0.24326(12)$ & $0.0559(5)$ \\
\hline $\mathrm{H} 7$ & 0.0897 & 0.2213 & 0.2223 & $0.067 *$ \\
\hline $\mathrm{C} 11$ & $0.2745(3)$ & $0.1148(2)$ & $-0.01380(14)$ & $0.0774(8)$ \\
\hline H11 & 0.2831 & 0.0581 & -0.0547 & $0.093 *$ \\
\hline $\mathrm{C} 6$ & $-0.0505(2)$ & $0.29260(19)$ & $0.29377(13)$ & $0.0628(6)$ \\
\hline H6 & -0.0876 & 0.2244 & 0.3059 & $0.075^{*}$ \\
\hline $\mathrm{C} 10$ & $0.1616(3)$ & $0.1723(2)$ & $0.00074(13)$ & $0.0687(7)$ \\
\hline $\mathrm{H} 10$ & 0.0930 & 0.1543 & -0.0305 & $0.082 *$ \\
\hline $\mathrm{C} 4$ & $-0.0455(2)$ & 0.49138 (19) & $0.30843(13)$ & $0.0605(6)$ \\
\hline $\mathrm{H} 4$ & -0.0793 & 0.5603 & 0.3304 & $0.073 *$ \\
\hline $\mathrm{C} 12$ & $0.3750(3)$ & $0.1417(2)$ & $0.03269(14)$ & $0.0727(7)$ \\
\hline H12 & 0.4521 & 0.1025 & 0.0235 & $0.087^{*}$ \\
\hline $\mathrm{C} 16$ & $0.4528(2)$ & $0.2656(2)$ & $0.34232(14)$ & $0.0753(7)$ \\
\hline H16 & 0.4639 & 0.1970 & 0.3722 & $0.090 *$ \\
\hline C19 & $0.4159(2)$ & $0.4675(2)$ & $0.25485(13)$ & $0.0666(6)$ \\
\hline H19 & 0.4030 & 0.5374 & 0.2271 & $0.080^{*}$ \\
\hline $\mathrm{C} 18$ & $0.5097(3)$ & $0.4537(3)$ & $0.31478(16)$ & $0.0946(10)$ \\
\hline H18 & 0.5600 & 0.5138 & 0.3255 & $0.113^{*}$ \\
\hline $\mathrm{C} 17$ & $0.5278(2)$ & $0.3528(3)$ & $0.35755(15)$ & $0.0896(9)$ \\
\hline H17 & 0.5909 & 0.3434 & 0.3969 & $0.107^{*}$ \\
\hline $\mathrm{O} 3$ & $0.83700(13)$ & 1.13075 (11) & $0.47955(8)$ & $0.0561(4)$ \\
\hline $\mathrm{C} 26$ & $0.77111(16)$ & $0.88371(15)$ & $0.33012(11)$ & 0.0409 (4) \\
\hline C39 & $0.88286(16)$ & $0.89755(15)$ & $0.26818(11)$ & $0.0414(4)$ \\
\hline $\mathrm{O} 4$ & $0.62945(13)$ & $1.09080(13)$ & $0.49852(9)$ & $0.0633(4)$ \\
\hline $\mathrm{C} 27$ & $0.65934(17)$ & $0.86683(15)$ & $0.27145(11)$ & $0.0426(4)$ \\
\hline $\mathrm{C} 45$ & $0.74962(16)$ & $0.99479(16)$ & $0.38537(11)$ & $0.0442(4)$ \\
\hline $\mathrm{H} 45$ & 0.7237 & 1.0581 & 0.3474 & $0.053^{*}$ \\
\hline $\mathrm{C} 40$ & $0.88986(19)$ & $0.99737(17)$ & $0.22169(12)$ & $0.0516(5)$ \\
\hline
\end{tabular}




\begin{tabular}{|c|c|c|c|c|}
\hline $\mathrm{H} 40$ & 0.8285 & 1.0562 & 0.2297 & $0.062 *$ \\
\hline $\mathrm{C} 33$ & $0.79058(18)$ & $0.78071(16)$ & $0.39142(11)$ & $0.0466(5)$ \\
\hline $\mathrm{C} 47$ & $0.86490(18)$ & $1.02937(16)$ & $0.43135(12)$ & $0.0493(5)$ \\
\hline H47A & 0.8942 & 0.9675 & 0.4686 & $0.059^{*}$ \\
\hline H47B & 0.9311 & 1.0427 & 0.3905 & $0.059 *$ \\
\hline $\mathrm{C} 46$ & $0.64654(18)$ & 0.98545 (19) & $0.45268(13)$ & $0.0571(5)$ \\
\hline $\mathrm{H} 46 \mathrm{~A}$ & 0.5690 & 0.9690 & 0.4260 & $0.069^{*}$ \\
\hline $\mathrm{H} 46 \mathrm{~B}$ & 0.6690 & 0.9231 & 0.4913 & $0.069^{*}$ \\
\hline $\mathrm{C} 29$ & $0.5612(2)$ & $0.7445(2)$ & $0.17364(13)$ & $0.0642(6)$ \\
\hline H29 & 0.5618 & 0.6753 & 0.1450 & $0.077^{*}$ \\
\hline $\mathrm{C} 28$ & $0.6577(2)$ & $0.76446(17)$ & $0.22676(12)$ & $0.0535(5)$ \\
\hline $\mathrm{H} 28$ & 0.7228 & 0.7089 & 0.2329 & $0.064 *$ \\
\hline C38 & $0.9023(2)$ & $0.75861(18)$ & $0.43297(12)$ & $0.0561(5)$ \\
\hline H38 & 0.9696 & 0.8023 & 0.4196 & $0.067 *$ \\
\hline $\mathrm{C} 44$ & $0.9728(2)$ & $0.81094(18)$ & $0.25085(13)$ & $0.0578(5)$ \\
\hline $\mathrm{H} 44$ & 0.9691 & 0.7411 & 0.2789 & $0.069^{*}$ \\
\hline $\mathrm{C} 41$ & $0.9853(2)$ & $1.01248(18)$ & $0.16365(13)$ & $0.0593(6)$ \\
\hline $\mathrm{H} 41$ & 0.9877 & 1.0810 & 0.1337 & $0.071 *$ \\
\hline $\mathrm{C} 43$ & $1.0682(2)$ & $0.8258(2)$ & $0.19260(14)$ & $0.0672(6)$ \\
\hline $\mathrm{H} 43$ & 1.1276 & 0.7660 & 0.1823 & $0.081^{*}$ \\
\hline $\mathrm{C} 42$ & $1.0762(2)$ & $0.92683(19)$ & $0.15016(13)$ & $0.0608(6)$ \\
\hline $\mathrm{H} 42$ & 1.1424 & 0.9375 & 0.1127 & $0.073 *$ \\
\hline $\mathrm{C} 32$ & $0.5633(2)$ & 0.94808 (19) & $0.25808(12)$ & $0.0587(5)$ \\
\hline $\mathrm{H} 32$ & 0.5631 & 1.0182 & 0.2854 & $0.070^{*}$ \\
\hline $\mathrm{C} 34$ & $0.6930(2)$ & $0.71336(18)$ & $0.41398(12)$ & $0.0578(5)$ \\
\hline H34 & 0.6164 & 0.7263 & 0.3880 & $0.069^{*}$ \\
\hline $\mathrm{C} 31$ & $0.4666(2)$ & $0.9269(2)$ & $0.20438(14)$ & $0.0721(7)$ \\
\hline $\mathrm{H} 31$ & 0.4021 & 0.9827 & 0.1968 & $0.087 *$ \\
\hline $\mathrm{C} 30$ & $0.4647(2)$ & $0.8250(2)$ & $0.16248(14)$ & $0.0706(7)$ \\
\hline $\mathrm{H} 30$ & 0.3992 & 0.8108 & 0.1272 & $0.085^{*}$ \\
\hline $\mathrm{C} 36$ & $0.8190(3)$ & $0.6071(2)$ & $0.51405(15)$ & $0.0844(8)$ \\
\hline H36 & 0.8289 & 0.5491 & 0.5543 & $0.101 *$ \\
\hline $\mathrm{C} 37$ & $0.9166(3)$ & $0.6740(2)$ & $0.49340(14)$ & $0.0753(7)$ \\
\hline H37 & 0.9925 & 0.6618 & 0.5205 & $0.090^{*}$ \\
\hline $\mathrm{C} 35$ & $0.7070(3)$ & $0.6273(2)$ & $0.47431(14)$ & $0.0750(7)$ \\
\hline H35 & 0.6403 & 0.5830 & 0.4879 & $0.090^{*}$ \\
\hline $\mathrm{C} 48$ & $0.73853(19)$ & $1.12060(19)$ & $0.54019(13)$ & $0.0584(6)$ \\
\hline $\mathrm{C} 23$ & $0.25244(18)$ & $0.63539(18)$ & $-0.03605(12)$ & $0.0533(5)$ \\
\hline $\mathrm{C} 49$ & $0.7751(2)$ & $1.0359(2)$ & $0.60968(14)$ & $0.0728(7)$ \\
\hline H49A & 0.7059 & 1.0295 & 0.6484 & $0.109 *$ \\
\hline H49B & 0.8451 & 1.0622 & 0.6393 & $0.109 *$ \\
\hline $\mathrm{H} 49 \mathrm{C}$ & 0.7975 & 0.9623 & 0.5854 & $0.109 *$ \\
\hline $\mathrm{C} 25$ & $0.2614(2)$ & $0.7575(2)$ & $-0.06423(17)$ & $0.0828(8)$ \\
\hline $\mathrm{H} 25 \mathrm{~A}$ & 0.2701 & 0.8051 & -0.0158 & $0.124 *$ \\
\hline $\mathrm{H} 25 \mathrm{~B}$ & 0.1871 & 0.7825 & -0.0937 & $0.124 *$ \\
\hline $\mathrm{H} 25 \mathrm{C}$ & 0.3328 & 0.7630 & -0.1012 & $0.124^{*}$ \\
\hline $\mathrm{C} 24$ & $0.2427(3)$ & $0.5554(2)$ & $-0.11037(13)$ & $0.0809(7)$ \\
\hline $\mathrm{H} 24 \mathrm{~A}$ & 0.3168 & 0.5573 & -0.1452 & $0.121 *$ \\
\hline
\end{tabular}




\begin{tabular}{lllll}
$\mathrm{H} 24 \mathrm{~B}$ & 0.1712 & 0.5796 & -0.1430 & $0.121^{*}$ \\
$\mathrm{H} 24 \mathrm{C}$ & 0.2339 & 0.4788 & -0.0900 & $0.121^{*}$ \\
$\mathrm{C} 50$ & $0.7094(2)$ & $1.2394(2)$ & $0.57462(16)$ & $0.0812(8)$ \\
$\mathrm{H} 50 \mathrm{~A}$ & 0.6878 & 1.2911 & 0.5291 & $0.122^{*}$ \\
$\mathrm{H} 50 \mathrm{~B}$ & 0.7814 & 1.2647 & 0.6025 & $0.122^{*}$ \\
$\mathrm{H} 50 \mathrm{C}$ & 0.6405 & 1.2382 & 0.6142 & $0.122^{*}$ \\
\hline
\end{tabular}

Atomic displacement parameters $\left(\AA^{2}\right)$

\begin{tabular}{|c|c|c|c|c|c|c|}
\hline & $U^{11}$ & $U^{22}$ & $U^{33}$ & $U^{12}$ & $U^{13}$ & $U^{23}$ \\
\hline $\mathrm{O} 1$ & $0.0381(7)$ & $0.0684(9)$ & $0.0581(8)$ & $-0.0039(6)$ & $-0.0018(6)$ & $0.0164(7)$ \\
\hline $\mathrm{O} 2$ & $0.0422(7)$ & $0.0641(9)$ & $0.0604(8)$ & $0.0094(6)$ & $0.0038(6)$ & $0.0162(7)$ \\
\hline $\mathrm{C} 1$ & $0.0353(9)$ & $0.0450(10)$ & $0.0387(9)$ & $-0.0001(7)$ & $-0.0037(7)$ & $-0.0040(8)$ \\
\hline $\mathrm{C} 20$ & $0.0343(9)$ & $0.0477(10)$ & $0.0406(9)$ & $-0.0013(8)$ & $-0.0018(7)$ & $-0.0016(8)$ \\
\hline $\mathrm{C} 2$ & $0.0416(10)$ & $0.0458(10)$ & $0.0359(9)$ & $-0.0038(8)$ & $-0.0026(7)$ & $-0.0009(8)$ \\
\hline $\mathrm{C} 14$ & $0.0411(10)$ & $0.0529(11)$ & $0.0393(9)$ & $0.0041(8)$ & $-0.0032(8)$ & $-0.0050(8)$ \\
\hline C8 & $0.0523(11)$ & $0.0433(10)$ & $0.0379(9)$ & $-0.0034(8)$ & $0.0014(8)$ & $-0.0046(8)$ \\
\hline $\mathrm{C} 22$ & $0.0365(10)$ & $0.0613(12)$ & $0.0495(11)$ & $0.0033(9)$ & $-0.0013(8)$ & $0.0096(9)$ \\
\hline C9 & $0.0586(13)$ & $0.0565(12)$ & $0.0487(11)$ & $-0.0149(10)$ & $-0.0026(9)$ & $-0.0047(9)$ \\
\hline $\mathrm{C} 21$ & $0.0358(10)$ & $0.0626(12)$ & $0.0543(11)$ & $-0.0014(9)$ & $-0.0027(8)$ & $0.0132(10)$ \\
\hline $\mathrm{C} 5$ & $0.0534(12)$ & $0.0716(14)$ & $0.0438(11)$ & $-0.0036(10)$ & $0.0080(9)$ & $0.0051(10)$ \\
\hline $\mathrm{C} 3$ & $0.0599(13)$ & $0.0456(11)$ & $0.0541(11)$ & $-0.0063(9)$ & $0.0131(10)$ & $-0.0037(9)$ \\
\hline $\mathrm{C} 13$ & $0.0608(13)$ & $0.0610(13)$ & $0.0500(11)$ & $0.0058(10)$ & $0.0011(10)$ & $-0.0096(10)$ \\
\hline $\mathrm{C} 15$ & $0.0651(14)$ & $0.0601(13)$ & $0.0528(12)$ & $0.0116(10)$ & $-0.0109(10)$ & $-0.0023(10)$ \\
\hline $\mathrm{C} 7$ & $0.0630(13)$ & $0.0496(12)$ & $0.0552(12)$ & $-0.0065(10)$ & $0.0076(10)$ & $-0.0024(9)$ \\
\hline C11 & $0.122(2)$ & $0.0614(15)$ & $0.0502(13)$ & -0.0180 & $0.0145(14)$ & -0.0183 \\
\hline C6 & $0.0662(14)$ & $0.0636(14)$ & $0.0600(13)$ & $-0.0201(11)$ & $0.0101(11)$ & $0.0029(11)$ \\
\hline $\mathrm{C} 10$ & $0.0908(19)$ & $0.0685(15)$ & $0.0502(12)$ & $-0.0308(14)$ & $-0.0022(12)$ & $-0.0122(11)$ \\
\hline $\mathrm{C} 4$ & $0.0671(14)$ & $0.0585(13)$ & $0.0541(12)$ & $0.0065(11)$ & $0.0136(10)$ & $-0.0037(10)$ \\
\hline $\mathrm{C} 12$ & $0.0911(18)$ & $0.0627(14)$ & $0.0613(14)$ & $0.0150(13)$ & $0.0144(13)$ & $-0.0119(12)$ \\
\hline $\mathrm{C} 16$ & $0.0769(17)$ & $0.0918(18)$ & $0.0531(13)$ & $0.0291(15)$ & $-0.0106(12)$ & $0.0094(13)$ \\
\hline C19 & $0.0656(14)$ & $0.0833(16)$ & $0.0538(12)$ & $-0.0241(12)$ & $-0.0184(11)$ & $0.0088(11)$ \\
\hline $\mathrm{C} 18$ & $0.0801(18)$ & $0.144(3)$ & $0.0663(16)$ & $-0.0493(18)$ & $-0.0329(14)$ & $0.0151(17)$ \\
\hline $\mathrm{C} 17$ & $0.0605(16)$ & $0.156(3)$ & $0.0525(14)$ & $-0.0076(17)$ & $-0.0195(12)$ & $0.0122(17)$ \\
\hline $\mathrm{O} 3$ & $0.0539(8)$ & $0.0572(8)$ & $0.0582(8)$ & $-0.0094(6)$ & $0.0013(7)$ & $-0.0184(7)$ \\
\hline $\mathrm{C} 26$ & $0.0394(10)$ & $0.0427(10)$ & $0.0406(9)$ & $-0.0018(8)$ & $-0.0012(7)$ & $-0.0035(8)$ \\
\hline C39 & $0.0398(10)$ & $0.0427(10)$ & $0.0420(9)$ & $-0.0046(8)$ & $-0.0009(8)$ & $-0.0058(8)$ \\
\hline $\mathrm{O} 4$ & $0.0431(8)$ & $0.0839(10)$ & $0.0627(9)$ & $0.0004(7)$ & $0.0009(6)$ & $-0.0299(8)$ \\
\hline $\mathrm{C} 27$ & $0.0412(10)$ & $0.0491(10)$ & $0.0383(9)$ & $-0.0082(8)$ & $-0.0007(8)$ & $0.0000(8)$ \\
\hline $\mathrm{C} 45$ & $0.0398(10)$ & 0.0494 (11) & $0.0433(10)$ & $-0.0008(8)$ & $-0.0002(8)$ & $-0.0076(8)$ \\
\hline $\mathrm{C} 40$ & $0.0551(12)$ & $0.0476(11)$ & $0.0519(11)$ & $-0.0030(9)$ & $0.0043(9)$ & $-0.0021(9)$ \\
\hline $\mathrm{C} 33$ & $0.0514(11)$ & $0.0475(11)$ & $0.0405(10)$ & $-0.0005(9)$ & $0.0007(8)$ & $-0.0029(8)$ \\
\hline $\mathrm{C} 47$ & $0.0423(10)$ & $0.0527(11)$ & $0.0534(11)$ & $-0.0062(8)$ & $0.0038(9)$ & $-0.0145(9)$ \\
\hline $\mathrm{C} 46$ & $0.0407(11)$ & $0.0751(14)$ & $0.0565(12)$ & $-0.0095(10)$ & $0.0034(9)$ & $-0.0248(10)$ \\
\hline $\mathrm{C} 29$ & $0.0742(16)$ & $0.0693(14)$ & $0.0521(12)$ & $-0.0245(12)$ & $-0.0074(11)$ & $-0.0092(11)$ \\
\hline $\mathrm{C} 28$ & $0.0598(13)$ & $0.0521(12)$ & $0.0498(11)$ & $-0.0116(10)$ & $-0.0029(9)$ & $-0.0037(9)$ \\
\hline $\mathrm{C} 38$ & $0.0605(13)$ & $0.0566(12)$ & $0.0508(11)$ & $0.0013(10)$ & $-0.0087(10)$ & $-0.0056(10)$ \\
\hline $\mathrm{C} 44$ & $0.0615(13)$ & $0.0482(11)$ & $0.0626(13)$ & $0.0028(10)$ & $0.0121(10)$ & $0.0009(10)$ \\
\hline
\end{tabular}


supporting information

$\begin{array}{lllllll}\text { C41 } & 0.0722(15) & 0.0529(12) & 0.0541(12) & -0.0156(11) & 0.0066(11) & 0.0014(10) \\ \text { C43 } & 0.0620(14) & 0.0647(14) & 0.0723(15) & 0.0099(11) & 0.0193(12) & -0.0033(12) \\ \text { C42 } & 0.0550(13) & 0.0701(15) & 0.0578(12) & -0.0125(11) & 0.0150(10) & -0.0062(11) \\ \text { C32 } & 0.0546(12) & 0.0692(14) & 0.0518(11) & 0.0035(11) & -0.0092(10) & -0.0106(10) \\ \text { C34 } & 0.0614(13) & 0.0649(13) & 0.0477(11) & -0.0104(11) & 0.0058(10) & 0.0008(10) \\ \text { C31 } & 0.0536(13) & 0.106(2) & 0.0555(13) & 0.0057(13) & -0.0110(10) & -0.0004(13) \\ \text { C30 } & 0.0582(14) & 0.105(2) & 0.0509(12) & -0.0175(14) & -0.0097(10) & -0.0061(13) \\ \text { C36 } & 0.131(3) & 0.0755(17) & 0.0456(13) & -0.0012(17) & -0.0040(15) & 0.0143(12) \\ \text { C37 } & 0.0946(19) & 0.0752(16) & 0.0547(13) & 0.0118(14) & -0.0221(13) & -0.0005(12) \\ \text { C35 } & 0.099(2) & 0.0758(16) & 0.0509(13) & -0.0183(14) & 0.0130(13) & 0.0066(12) \\ \text { C48 } & 0.0465(11) & 0.0760(15) & 0.0531(12) & -0.0049(10) & 0.0003(9) & -0.0229(11) \\ \text { C23 } & 0.0398(11) & 0.0672(13) & 0.0519(11) & 0.0037(9) & 0.0009(9) & 0.0150(10) \\ \text { C49 } & 0.0682(15) & 0.0963(18) & 0.0542(13) & -0.0077(13) & 0.0022(11) & -0.0114(13) \\ \text { C25 } & 0.0684(16) & 0.0839(18) & 0.0941(18) & 0.0031(13) & 0.0052(14) & 0.0387(15) \\ \text { C24 } & 0.0796(17) & 0.114(2) & 0.0469(12) & 0.0107(15) & -0.0044(12) & 0.0026(13) \\ \text { C50 } & 0.0734(17) & 0.0898(18) & 0.0798(16) & 0.0063(13) & -0.0030(13) & -0.0411(14) \\ & & & & & \end{array}$

Geometric parameters $\left(\AA,{ }^{\circ}\right)$

\begin{tabular}{llll}
\hline $\mathrm{O} 1-\mathrm{C} 23$ & $1.420(2)$ & $\mathrm{O} 4-\mathrm{C} 48$ & $1.417(2)$ \\
$\mathrm{O} 1-\mathrm{C} 22$ & $1.430(2)$ & $\mathrm{O} 4-\mathrm{C} 46$ & $1.435(2)$ \\
$\mathrm{O} 2-\mathrm{C} 21$ & $1.420(2)$ & $\mathrm{C} 27-\mathrm{C} 32$ & $1.376(3)$ \\
$\mathrm{O} 2-\mathrm{C} 23$ & $1.428(2)$ & $\mathrm{C} 27-\mathrm{C} 28$ & $1.396(3)$ \\
$\mathrm{C} 1-\mathrm{C} 2$ & $1.546(2)$ & $\mathrm{C} 45-\mathrm{C} 47$ & $1.524(2)$ \\
$\mathrm{C} 1-\mathrm{C} 8$ & $1.547(2)$ & $\mathrm{C} 45-\mathrm{C} 46$ & $1.529(3)$ \\
$\mathrm{C} 1-\mathrm{C} 14$ & $1.554(2)$ & $\mathrm{C} 45-\mathrm{H} 45$ & 0.9800 \\
$\mathrm{C} 1-\mathrm{C} 20$ & $1.565(2)$ & $\mathrm{C} 40-\mathrm{C} 41$ & $1.381(3)$ \\
$\mathrm{C} 20-\mathrm{C} 21$ & $1.523(2)$ & $\mathrm{C} 40-\mathrm{H} 40$ & 0.9300 \\
$\mathrm{C} 20-\mathrm{C} 22$ & $1.528(2)$ & $\mathrm{C} 33-\mathrm{C} 38$ & $1.388(3)$ \\
$\mathrm{C} 20-\mathrm{H} 20$ & 0.9800 & $\mathrm{C} 33-\mathrm{C} 34$ & $1.388(3)$ \\
$\mathrm{C} 2-\mathrm{C} 3$ & $1.383(3)$ & $\mathrm{C} 47-\mathrm{H} 47 \mathrm{~A}$ & 0.9700 \\
$\mathrm{C} 2-\mathrm{C} 7$ & $1.384(3)$ & $\mathrm{C} 47-\mathrm{H} 47 \mathrm{~B}$ & 0.9700 \\
$\mathrm{C} 14-\mathrm{C} 19$ & $1.379(3)$ & $\mathrm{C} 46-\mathrm{H} 46 \mathrm{~A}$ & 0.9700 \\
$\mathrm{C} 14-\mathrm{C} 15$ & $1.391(3)$ & $\mathrm{C} 46-\mathrm{H} 46 \mathrm{~B}$ & 0.9700 \\
$\mathrm{C} 8-\mathrm{C} 13$ & $1.383(3)$ & $\mathrm{C} 29-\mathrm{C} 30$ & $1.368(3)$ \\
$\mathrm{C} 8-\mathrm{C} 9$ & $\mathrm{C} 29-\mathrm{C} 28$ & $1.378(3)$ \\
$\mathrm{C} 22-\mathrm{H} 22 \mathrm{~A}$ & $1.391(3)$ & $\mathrm{C} 29-\mathrm{H} 29$ & 0.9300 \\
$\mathrm{C} 22-\mathrm{H} 22 \mathrm{~B}$ & 0.9700 & $\mathrm{C} 28-\mathrm{H} 28$ & 0.9300 \\
$\mathrm{C} 9-\mathrm{C} 10$ & 0.9700 & $\mathrm{C} 38-\mathrm{C} 37$ & $1.375(3)$ \\
$\mathrm{C} 9-\mathrm{H} 9$ & $1.382(3)$ & $\mathrm{C} 38-\mathrm{H} 38$ & 0.9300 \\
$\mathrm{C} 21-\mathrm{H} 21 \mathrm{~A}$ & 0.9300 & $\mathrm{C} 44-\mathrm{C} 43$ & $1.383(3)$ \\
$\mathrm{C} 21-\mathrm{H} 21 \mathrm{~B}$ & 0.9700 & $\mathrm{C} 44-\mathrm{H} 44$ & 0.9300 \\
$\mathrm{C} 5-\mathrm{C} 6$ & 0.9700 & $\mathrm{C} 41-\mathrm{C} 42$ & $1.369(3)$ \\
$\mathrm{C} 5-\mathrm{C} 4$ & $1.360(3)$ & $\mathrm{C} 41-\mathrm{H} 41$ & 0.9300 \\
$\mathrm{C} 5-\mathrm{H} 5$ & $1.361(3)$ & $\mathrm{C} 43-\mathrm{C} 42$ & $1.361(3)$ \\
$\mathrm{C} 3-\mathrm{C} 4$ & $\mathrm{C} 42-\mathrm{H} 43$ & 0.9300 \\
$\mathrm{C} 3-\mathrm{H} 32$ & $\mathrm{C} 32-\mathrm{C} 31$ & $1.389(3)$ \\
$\mathrm{C} 13-\mathrm{C} 12$ & $1.384(3)$ & &
\end{tabular}




\begin{tabular}{|c|c|c|c|}
\hline C13-H13 & 0.9300 & $\mathrm{C} 32-\mathrm{H} 32$ & 0.9300 \\
\hline $\mathrm{C} 15-\mathrm{C} 16$ & $1.378(3)$ & $\mathrm{C} 34-\mathrm{C} 35$ & $1.386(3)$ \\
\hline C15-H15 & 0.9300 & C34-H34 & 0.9300 \\
\hline $\mathrm{C} 7-\mathrm{C} 6$ & $1.383(3)$ & $\mathrm{C} 31-\mathrm{C} 30$ & $1.369(3)$ \\
\hline $\mathrm{C} 7-\mathrm{H} 7$ & 0.9300 & $\mathrm{C} 31-\mathrm{H} 31$ & 0.9300 \\
\hline $\mathrm{C} 11-\mathrm{C} 10$ & $1.364(4)$ & $\mathrm{C} 30-\mathrm{H} 30$ & 0.9300 \\
\hline $\mathrm{C} 11-\mathrm{C} 12$ & $1.370(4)$ & $\mathrm{C} 36-\mathrm{C} 35$ & $1.374(4)$ \\
\hline C11-H11 & 0.9300 & $\mathrm{C} 36-\mathrm{C} 37$ & $1.377(4)$ \\
\hline $\mathrm{C} 6-\mathrm{H} 6$ & 0.9300 & C36-H36 & 0.9300 \\
\hline $\mathrm{C} 10-\mathrm{H} 10$ & 0.9300 & C37-H37 & 0.9300 \\
\hline $\mathrm{C} 4-\mathrm{H} 4$ & 0.9300 & $\mathrm{C} 35-\mathrm{H} 35$ & 0.9300 \\
\hline $\mathrm{C} 12-\mathrm{H} 12$ & 0.9300 & $\mathrm{C} 48-\mathrm{C} 50$ & $1.509(3)$ \\
\hline $\mathrm{C} 16-\mathrm{C} 17$ & $1.363(4)$ & $\mathrm{C} 48-\mathrm{C} 49$ & $1.516(3)$ \\
\hline $\mathrm{C} 16-\mathrm{H} 16$ & 0.9300 & $\mathrm{C} 23-\mathrm{C} 25$ & $1.501(3)$ \\
\hline $\mathrm{C} 19-\mathrm{C} 18$ & $1.398(3)$ & $\mathrm{C} 23-\mathrm{C} 24$ & $1.518(3)$ \\
\hline C19-H19 & 0.9300 & $\mathrm{C} 49-\mathrm{H} 49 \mathrm{~A}$ & 0.9600 \\
\hline $\mathrm{C} 18-\mathrm{C} 17$ & $1.362(4)$ & $\mathrm{C} 49-\mathrm{H} 49 \mathrm{~B}$ & 0.9600 \\
\hline $\mathrm{C} 18-\mathrm{H} 18$ & 0.9300 & $\mathrm{C} 49-\mathrm{H} 49 \mathrm{C}$ & 0.9600 \\
\hline C17- $\mathrm{H} 17$ & 0.9300 & $\mathrm{C} 25-\mathrm{H} 25 \mathrm{~A}$ & 0.9600 \\
\hline $\mathrm{O} 3-\mathrm{C} 48$ & $1.424(2)$ & $\mathrm{C} 25-\mathrm{H} 25 \mathrm{~B}$ & 0.9600 \\
\hline $\mathrm{O} 3-\mathrm{C} 47$ & $1.428(2)$ & $\mathrm{C} 25-\mathrm{H} 25 \mathrm{C}$ & 0.9600 \\
\hline $\mathrm{C} 26-\mathrm{C} 33$ & $1.548(3)$ & $\mathrm{C} 24-\mathrm{H} 24 \mathrm{~A}$ & 0.9600 \\
\hline $\mathrm{C} 26-\mathrm{C} 39$ & $1.550(2)$ & $\mathrm{C} 24-\mathrm{H} 24 \mathrm{~B}$ & 0.9600 \\
\hline $\mathrm{C} 26-\mathrm{C} 27$ & $1.554(2)$ & $\mathrm{C} 24-\mathrm{H} 24 \mathrm{C}$ & 0.9600 \\
\hline $\mathrm{C} 26-\mathrm{C} 45$ & $1.574(2)$ & $\mathrm{C} 50-\mathrm{H} 50 \mathrm{~A}$ & 0.9600 \\
\hline $\mathrm{C} 39-\mathrm{C} 44$ & $1.379(3)$ & $\mathrm{C} 50-\mathrm{H} 50 \mathrm{~B}$ & 0.9600 \\
\hline $\mathrm{C} 39-\mathrm{C} 40$ & $1.381(2)$ & $\mathrm{C} 50-\mathrm{H} 50 \mathrm{C}$ & 0.9600 \\
\hline $\mathrm{C} 23-\mathrm{O} 1-\mathrm{C} 22$ & $114.18(14)$ & $\mathrm{C} 46-\mathrm{C} 45-\mathrm{H} 45$ & 107.5 \\
\hline $\mathrm{C} 21-\mathrm{O} 2-\mathrm{C} 23$ & $114.44(14)$ & $\mathrm{C} 26-\mathrm{C} 45-\mathrm{H} 45$ & 107.5 \\
\hline $\mathrm{C} 2-\mathrm{C} 1-\mathrm{C} 8$ & $112.14(14)$ & $\mathrm{C} 39-\mathrm{C} 40-\mathrm{C} 41$ & $122.00(19)$ \\
\hline $\mathrm{C} 2-\mathrm{C} 1-\mathrm{C} 14$ & $104.69(13)$ & $\mathrm{C} 39-\mathrm{C} 40-\mathrm{H} 40$ & 119.0 \\
\hline $\mathrm{C} 8-\mathrm{C} 1-\mathrm{C} 14$ & $110.69(14)$ & $\mathrm{C} 41-\mathrm{C} 40-\mathrm{H} 40$ & 119.0 \\
\hline $\mathrm{C} 2-\mathrm{C} 1-\mathrm{C} 20$ & $111.03(14)$ & $\mathrm{C} 38-\mathrm{C} 33-\mathrm{C} 34$ & $116.75(18)$ \\
\hline $\mathrm{C} 8-\mathrm{C} 1-\mathrm{C} 20$ & $107.10(13)$ & $\mathrm{C} 38-\mathrm{C} 33-\mathrm{C} 26$ & $121.56(17)$ \\
\hline $\mathrm{C} 14-\mathrm{C} 1-\mathrm{C} 20$ & $111.27(14)$ & $\mathrm{C} 34-\mathrm{C} 33-\mathrm{C} 26$ & $121.36(18)$ \\
\hline $\mathrm{C} 21-\mathrm{C} 20-\mathrm{C} 22$ & $106.52(14)$ & $\mathrm{O} 3-\mathrm{C} 47-\mathrm{C} 45$ & $110.77(15)$ \\
\hline $\mathrm{C} 21-\mathrm{C} 20-\mathrm{C} 1$ & $115.43(14)$ & $\mathrm{O} 3-\mathrm{C} 47-\mathrm{H} 47 \mathrm{~A}$ & 109.5 \\
\hline $\mathrm{C} 22-\mathrm{C} 20-\mathrm{C} 1$ & $113.94(14)$ & $\mathrm{C} 45-\mathrm{C} 47-\mathrm{H} 47 \mathrm{~A}$ & 109.5 \\
\hline $\mathrm{C} 21-\mathrm{C} 20-\mathrm{H} 20$ & 106.8 & $\mathrm{O} 3-\mathrm{C} 47-\mathrm{H} 47 \mathrm{~B}$ & 109.5 \\
\hline $\mathrm{C} 22-\mathrm{C} 20-\mathrm{H} 20$ & 106.8 & $\mathrm{C} 45-\mathrm{C} 47-\mathrm{H} 47 \mathrm{~B}$ & 109.5 \\
\hline $\mathrm{C} 1-\mathrm{C} 20-\mathrm{H} 20$ & 106.8 & $\mathrm{H} 47 \mathrm{~A}-\mathrm{C} 47-\mathrm{H} 47 \mathrm{~B}$ & 108.1 \\
\hline $\mathrm{C} 3-\mathrm{C} 2-\mathrm{C} 7$ & $116.45(17)$ & $\mathrm{O} 4-\mathrm{C} 46-\mathrm{C} 45$ & $109.95(16)$ \\
\hline $\mathrm{C} 3-\mathrm{C} 2-\mathrm{C} 1$ & $121.60(16)$ & $\mathrm{O} 4-\mathrm{C} 46-\mathrm{H} 46 \mathrm{~A}$ & 109.7 \\
\hline $\mathrm{C} 7-\mathrm{C} 2-\mathrm{C} 1$ & $121.77(16)$ & $\mathrm{C} 45-\mathrm{C} 46-\mathrm{H} 46 \mathrm{~A}$ & 109.7 \\
\hline $\mathrm{C} 19-\mathrm{C} 14-\mathrm{C} 15$ & $117.11(18)$ & $\mathrm{O} 4-\mathrm{C} 46-\mathrm{H} 46 \mathrm{~B}$ & 109.7 \\
\hline $\mathrm{C} 19-\mathrm{C} 14-\mathrm{C} 1$ & $123.60(18)$ & $\mathrm{C} 45-\mathrm{C} 46-\mathrm{H} 46 \mathrm{~B}$ & 109.7 \\
\hline $\mathrm{C} 15-\mathrm{C} 14-\mathrm{C} 1$ & $119.21(17)$ & $\mathrm{H} 46 \mathrm{~A}-\mathrm{C} 46-\mathrm{H} 46 \mathrm{~B}$ & 108.2 \\
\hline
\end{tabular}




\begin{tabular}{|c|c|c|c|}
\hline $\mathrm{C} 13-\mathrm{C} 8-\mathrm{C} 9$ & $117.53(17)$ & $\mathrm{C} 30-\mathrm{C} 29-\mathrm{C} 28$ & $120.9(2)$ \\
\hline $\mathrm{C} 13-\mathrm{C} 8-\mathrm{C} 1$ & $121.48(17)$ & $\mathrm{C} 30-\mathrm{C} 29-\mathrm{H} 29$ & 119.5 \\
\hline $\mathrm{C} 9-\mathrm{C} 8-\mathrm{C} 1$ & $120.75(17)$ & $\mathrm{C} 28-\mathrm{C} 29-\mathrm{H} 29$ & 119.5 \\
\hline $\mathrm{O} 1-\mathrm{C} 22-\mathrm{C} 20$ & $110.11(15)$ & $\mathrm{C} 29-\mathrm{C} 28-\mathrm{C} 27$ & $121.1(2)$ \\
\hline $\mathrm{O} 1-\mathrm{C} 22-\mathrm{H} 22 \mathrm{~A}$ & 109.6 & $\mathrm{C} 29-\mathrm{C} 28-\mathrm{H} 28$ & 119.5 \\
\hline $\mathrm{C} 20-\mathrm{C} 22-\mathrm{H} 22 \mathrm{~A}$ & 109.6 & $\mathrm{C} 27-\mathrm{C} 28-\mathrm{H} 28$ & 119.5 \\
\hline $\mathrm{O} 1-\mathrm{C} 22-\mathrm{H} 22 \mathrm{~B}$ & 109.6 & $\mathrm{C} 37-\mathrm{C} 38-\mathrm{C} 33$ & $121.9(2)$ \\
\hline $\mathrm{C} 20-\mathrm{C} 22-\mathrm{H} 22 \mathrm{~B}$ & 109.6 & $\mathrm{C} 37-\mathrm{C} 38-\mathrm{H} 38$ & 119.0 \\
\hline $\mathrm{H} 22 \mathrm{~A}-\mathrm{C} 22-\mathrm{H} 22 \mathrm{~B}$ & 108.2 & $\mathrm{C} 33-\mathrm{C} 38-\mathrm{H} 38$ & 119.0 \\
\hline $\mathrm{C} 10-\mathrm{C} 9-\mathrm{C} 8$ & $120.9(2)$ & $\mathrm{C} 39-\mathrm{C} 44-\mathrm{C} 43$ & $121.4(2)$ \\
\hline $\mathrm{C} 10-\mathrm{C} 9-\mathrm{H} 9$ & 119.6 & $\mathrm{C} 39-\mathrm{C} 44-\mathrm{H} 44$ & 119.3 \\
\hline $\mathrm{C} 8-\mathrm{C} 9-\mathrm{H} 9$ & 119.6 & $\mathrm{C} 43-\mathrm{C} 44-\mathrm{H} 44$ & 119.3 \\
\hline $\mathrm{O} 2-\mathrm{C} 21-\mathrm{C} 20$ & $110.71(15)$ & $\mathrm{C} 42-\mathrm{C} 41-\mathrm{C} 40$ & $120.2(2)$ \\
\hline $\mathrm{O} 2-\mathrm{C} 21-\mathrm{H} 21 \mathrm{~A}$ & 109.5 & $\mathrm{C} 42-\mathrm{C} 41-\mathrm{H} 41$ & 119.9 \\
\hline $\mathrm{C} 20-\mathrm{C} 21-\mathrm{H} 21 \mathrm{~A}$ & 109.5 & $\mathrm{C} 40-\mathrm{C} 41-\mathrm{H} 41$ & 119.9 \\
\hline $\mathrm{O} 2-\mathrm{C} 21-\mathrm{H} 21 \mathrm{~B}$ & 109.5 & $\mathrm{C} 42-\mathrm{C} 43-\mathrm{C} 44$ & $120.9(2)$ \\
\hline $\mathrm{C} 20-\mathrm{C} 21-\mathrm{H} 21 \mathrm{~B}$ & 109.5 & $\mathrm{C} 42-\mathrm{C} 43-\mathrm{H} 43$ & 119.6 \\
\hline $\mathrm{H} 21 \mathrm{~A}-\mathrm{C} 21-\mathrm{H} 21 \mathrm{~B}$ & 108.1 & $\mathrm{C} 44-\mathrm{C} 43-\mathrm{H} 43$ & 119.6 \\
\hline $\mathrm{C} 6-\mathrm{C} 5-\mathrm{C} 4$ & $118.78(19)$ & $\mathrm{C} 43-\mathrm{C} 42-\mathrm{C} 41$ & $118.82(19)$ \\
\hline $\mathrm{C} 6-\mathrm{C} 5-\mathrm{H} 5$ & 120.6 & $\mathrm{C} 43-\mathrm{C} 42-\mathrm{H} 42$ & 120.6 \\
\hline $\mathrm{C} 4-\mathrm{C} 5-\mathrm{H} 5$ & 120.6 & $\mathrm{C} 41-\mathrm{C} 42-\mathrm{H} 42$ & 120.6 \\
\hline $\mathrm{C} 2-\mathrm{C} 3-\mathrm{C} 4$ & $121.57(18)$ & $\mathrm{C} 27-\mathrm{C} 32-\mathrm{C} 31$ & $120.9(2)$ \\
\hline $\mathrm{C} 2-\mathrm{C} 3-\mathrm{H} 3$ & 119.2 & $\mathrm{C} 27-\mathrm{C} 32-\mathrm{H} 32$ & 119.5 \\
\hline $\mathrm{C} 4-\mathrm{C} 3-\mathrm{H} 3$ & 119.2 & $\mathrm{C} 31-\mathrm{C} 32-\mathrm{H} 32$ & 119.5 \\
\hline $\mathrm{C} 8-\mathrm{C} 13-\mathrm{C} 12$ & $121.1(2)$ & $\mathrm{C} 35-\mathrm{C} 34-\mathrm{C} 33$ & $121.6(2)$ \\
\hline $\mathrm{C} 8-\mathrm{C} 13-\mathrm{H} 13$ & 119.5 & $\mathrm{C} 35-\mathrm{C} 34-\mathrm{H} 34$ & 119.2 \\
\hline $\mathrm{C} 12-\mathrm{C} 13-\mathrm{H} 13$ & 119.5 & $\mathrm{C} 33-\mathrm{C} 34-\mathrm{H} 34$ & 119.2 \\
\hline $\mathrm{C} 16-\mathrm{C} 15-\mathrm{C} 14$ & $121.5(2)$ & $\mathrm{C} 30-\mathrm{C} 31-\mathrm{C} 32$ & $121.0(2)$ \\
\hline $\mathrm{C} 16-\mathrm{C} 15-\mathrm{H} 15$ & 119.3 & $\mathrm{C} 30-\mathrm{C} 31-\mathrm{H} 31$ & 119.5 \\
\hline $\mathrm{C} 14-\mathrm{C} 15-\mathrm{H} 15$ & 119.3 & $\mathrm{C} 32-\mathrm{C} 31-\mathrm{H} 31$ & 119.5 \\
\hline $\mathrm{C} 6-\mathrm{C} 7-\mathrm{C} 2$ & $121.51(19)$ & $\mathrm{C} 29-\mathrm{C} 30-\mathrm{C} 31$ & $118.6(2)$ \\
\hline $\mathrm{C} 6-\mathrm{C} 7-\mathrm{H} 7$ & 119.2 & $\mathrm{C} 29-\mathrm{C} 30-\mathrm{H} 30$ & 120.7 \\
\hline $\mathrm{C} 2-\mathrm{C} 7-\mathrm{H} 7$ & 119.2 & $\mathrm{C} 31-\mathrm{C} 30-\mathrm{H} 30$ & 120.7 \\
\hline $\mathrm{C} 10-\mathrm{C} 11-\mathrm{C} 12$ & $119.2(2)$ & $\mathrm{C} 35-\mathrm{C} 36-\mathrm{C} 37$ & $119.0(2)$ \\
\hline $\mathrm{C} 10-\mathrm{C} 11-\mathrm{H} 11$ & 120.4 & $\mathrm{C} 35-\mathrm{C} 36-\mathrm{H} 36$ & 120.5 \\
\hline $\mathrm{C} 12-\mathrm{C} 11-\mathrm{H} 11$ & 120.4 & $\mathrm{C} 37-\mathrm{C} 36-\mathrm{H} 36$ & 120.5 \\
\hline $\mathrm{C} 5-\mathrm{C} 6-\mathrm{C} 7$ & $120.88(19)$ & $\mathrm{C} 38-\mathrm{C} 37-\mathrm{C} 36$ & $120.4(2)$ \\
\hline $\mathrm{C} 5-\mathrm{C} 6-\mathrm{H} 6$ & 119.6 & $\mathrm{C} 38-\mathrm{C} 37-\mathrm{H} 37$ & 119.8 \\
\hline $\mathrm{C} 7-\mathrm{C} 6-\mathrm{H} 6$ & 119.6 & $\mathrm{C} 36-\mathrm{C} 37-\mathrm{H} 37$ & 119.8 \\
\hline $\mathrm{C} 11-\mathrm{C} 10-\mathrm{C} 9$ & $120.8(2)$ & $\mathrm{C} 36-\mathrm{C} 35-\mathrm{C} 34$ & $120.3(2)$ \\
\hline $\mathrm{C} 11-\mathrm{C} 10-\mathrm{H} 10$ & 119.6 & $\mathrm{C} 36-\mathrm{C} 35-\mathrm{H} 35$ & 119.9 \\
\hline $\mathrm{C} 9-\mathrm{C} 10-\mathrm{H} 10$ & 119.6 & $\mathrm{C} 34-\mathrm{C} 35-\mathrm{H} 35$ & 119.9 \\
\hline $\mathrm{C} 5-\mathrm{C} 4-\mathrm{C} 3$ & $120.77(19)$ & $\mathrm{O} 4-\mathrm{C} 48-\mathrm{O} 3$ & $109.22(15)$ \\
\hline $\mathrm{C} 5-\mathrm{C} 4-\mathrm{H} 4$ & 119.6 & $\mathrm{O} 4-\mathrm{C} 48-\mathrm{C} 50$ & $106.01(17)$ \\
\hline $\mathrm{C} 3-\mathrm{C} 4-\mathrm{H} 4$ & 119.6 & $\mathrm{O} 3-\mathrm{C} 48-\mathrm{C} 50$ & $106.01(18)$ \\
\hline $\mathrm{C} 11-\mathrm{C} 12-\mathrm{C} 13$ & $120.6(2)$ & $\mathrm{O} 4-\mathrm{C} 48-\mathrm{C} 49$ & $111.97(19)$ \\
\hline $\mathrm{C} 11-\mathrm{C} 12-\mathrm{H} 12$ & 119.7 & $\mathrm{O} 3-\mathrm{C} 48-\mathrm{C} 49$ & $111.75(18)$ \\
\hline $\mathrm{C} 13-\mathrm{C} 12-\mathrm{H} 12$ & 119.7 & $\mathrm{C} 50-\mathrm{C} 48-\mathrm{C} 49$ & $111.56(18)$ \\
\hline
\end{tabular}




\begin{tabular}{|c|c|c|c|}
\hline $\mathrm{C} 17-\mathrm{C} 16-\mathrm{C} 15$ & $120.5(2)$ & $\mathrm{O} 1-\mathrm{C} 23-\mathrm{O} 2$ & $109.10(15)$ \\
\hline $\mathrm{C} 17-\mathrm{C} 16-\mathrm{H} 16$ & 119.8 & $\mathrm{O} 1-\mathrm{C} 23-\mathrm{C} 25$ & $106.39(17)$ \\
\hline $\mathrm{C} 15-\mathrm{C} 16-\mathrm{H} 16$ & 119.8 & $\mathrm{O} 2-\mathrm{C} 23-\mathrm{C} 25$ & $105.74(17)$ \\
\hline $\mathrm{C} 14-\mathrm{C} 19-\mathrm{C} 18$ & $121.0(2)$ & $\mathrm{O} 1-\mathrm{C} 23-\mathrm{C} 24$ & $112.28(17)$ \\
\hline $\mathrm{C} 14-\mathrm{C} 19-\mathrm{H} 19$ & 119.5 & $\mathrm{O} 2-\mathrm{C} 23-\mathrm{C} 24$ & $111.23(18)$ \\
\hline $\mathrm{C} 18-\mathrm{C} 19-\mathrm{H} 19$ & 119.5 & $\mathrm{C} 25-\mathrm{C} 23-\mathrm{C} 24$ & $111.78(19)$ \\
\hline $\mathrm{C} 17-\mathrm{C} 18-\mathrm{C} 19$ & $120.2(2)$ & $\mathrm{C} 48-\mathrm{C} 49-\mathrm{H} 49 \mathrm{~A}$ & 109.5 \\
\hline $\mathrm{C} 17-\mathrm{C} 18-\mathrm{H} 18$ & 119.9 & $\mathrm{C} 48-\mathrm{C} 49-\mathrm{H} 49 \mathrm{~B}$ & 109.5 \\
\hline $\mathrm{C} 19-\mathrm{C} 18-\mathrm{H} 18$ & 119.9 & $\mathrm{H} 49 \mathrm{~A}-\mathrm{C} 49-\mathrm{H} 49 \mathrm{~B}$ & 109.5 \\
\hline $\mathrm{C} 18-\mathrm{C} 17-\mathrm{C} 16$ & $119.6(2)$ & $\mathrm{C} 48-\mathrm{C} 49-\mathrm{H} 49 \mathrm{C}$ & 109.5 \\
\hline $\mathrm{C} 18-\mathrm{C} 17-\mathrm{H} 17$ & 120.2 & $\mathrm{H} 49 \mathrm{~A}-\mathrm{C} 49-\mathrm{H} 49 \mathrm{C}$ & 109.5 \\
\hline $\mathrm{C} 16-\mathrm{C} 17-\mathrm{H} 17$ & 120.2 & $\mathrm{H} 49 \mathrm{~B}-\mathrm{C} 49-\mathrm{H} 49 \mathrm{C}$ & 109.5 \\
\hline $\mathrm{C} 48-\mathrm{O} 3-\mathrm{C} 47$ & $113.78(14)$ & $\mathrm{C} 23-\mathrm{C} 25-\mathrm{H} 25 \mathrm{~A}$ & 109.5 \\
\hline $\mathrm{C} 33-\mathrm{C} 26-\mathrm{C} 39$ & $113.51(14)$ & $\mathrm{C} 23-\mathrm{C} 25-\mathrm{H} 25 \mathrm{~B}$ & 109.5 \\
\hline $\mathrm{C} 33-\mathrm{C} 26-\mathrm{C} 27$ & $110.61(14)$ & $\mathrm{H} 25 \mathrm{~A}-\mathrm{C} 25-\mathrm{H} 25 \mathrm{~B}$ & 109.5 \\
\hline $\mathrm{C} 39-\mathrm{C} 26-\mathrm{C} 27$ & $103.98(13)$ & $\mathrm{C} 23-\mathrm{C} 25-\mathrm{H} 25 \mathrm{C}$ & 109.5 \\
\hline $\mathrm{C} 33-\mathrm{C} 26-\mathrm{C} 45$ & $107.38(14)$ & $\mathrm{H} 25 \mathrm{~A}-\mathrm{C} 25-\mathrm{H} 25 \mathrm{C}$ & 109.5 \\
\hline $\mathrm{C} 39-\mathrm{C} 26-\mathrm{C} 45$ & $109.49(13)$ & $\mathrm{H} 25 \mathrm{~B}-\mathrm{C} 25-\mathrm{H} 25 \mathrm{C}$ & 109.5 \\
\hline $\mathrm{C} 27-\mathrm{C} 26-\mathrm{C} 45$ & $111.94(14)$ & $\mathrm{C} 23-\mathrm{C} 24-\mathrm{H} 24 \mathrm{~A}$ & 109.5 \\
\hline $\mathrm{C} 44-\mathrm{C} 39-\mathrm{C} 40$ & $116.61(17)$ & $\mathrm{C} 23-\mathrm{C} 24-\mathrm{H} 24 \mathrm{~B}$ & 109.5 \\
\hline $\mathrm{C} 44-\mathrm{C} 39-\mathrm{C} 26$ & $123.42(17)$ & $\mathrm{H} 24 \mathrm{~A}-\mathrm{C} 24-\mathrm{H} 24 \mathrm{~B}$ & 109.5 \\
\hline $\mathrm{C} 40-\mathrm{C} 39-\mathrm{C} 26$ & $119.74(16)$ & $\mathrm{C} 23-\mathrm{C} 24-\mathrm{H} 24 \mathrm{C}$ & 109.5 \\
\hline $\mathrm{C} 48-\mathrm{O} 4-\mathrm{C} 46$ & $113.22(15)$ & $\mathrm{H} 24 \mathrm{~A}-\mathrm{C} 24-\mathrm{H} 24 \mathrm{C}$ & 109.5 \\
\hline $\mathrm{C} 32-\mathrm{C} 27-\mathrm{C} 28$ & $117.37(18)$ & $\mathrm{H} 24 \mathrm{~B}-\mathrm{C} 24-\mathrm{H} 24 \mathrm{C}$ & 109.5 \\
\hline $\mathrm{C} 32-\mathrm{C} 27-\mathrm{C} 26$ & $124.24(16)$ & $\mathrm{C} 48-\mathrm{C} 50-\mathrm{H} 50 \mathrm{~A}$ & 109.5 \\
\hline $\mathrm{C} 28-\mathrm{C} 27-\mathrm{C} 26$ & $118.35(17)$ & $\mathrm{C} 48-\mathrm{C} 50-\mathrm{H} 50 \mathrm{~B}$ & 109.5 \\
\hline $\mathrm{C} 47-\mathrm{C} 45-\mathrm{C} 46$ & $106.41(14)$ & $\mathrm{H} 50 \mathrm{~A}-\mathrm{C} 50-\mathrm{H} 50 \mathrm{~B}$ & 109.5 \\
\hline $\mathrm{C} 47-\mathrm{C} 45-\mathrm{C} 26$ & $114.60(14)$ & $\mathrm{C} 48-\mathrm{C} 50-\mathrm{H} 50 \mathrm{C}$ & 109.5 \\
\hline $\mathrm{C} 46-\mathrm{C} 45-\mathrm{C} 26$ & $113.10(15)$ & $\mathrm{H} 50 \mathrm{~A}-\mathrm{C} 50-\mathrm{H} 50 \mathrm{C}$ & 109.5 \\
\hline $\mathrm{C} 47-\mathrm{C} 45-\mathrm{H} 45$ & 107.5 & $\mathrm{H} 50 \mathrm{~B}-\mathrm{C} 50-\mathrm{H} 50 \mathrm{C}$ & 109.5 \\
\hline $\mathrm{C} 2-\mathrm{C} 1-\mathrm{C} 20-\mathrm{C} 21$ & $-51.71(19)$ & $\mathrm{C} 33-\mathrm{C} 26-\mathrm{C} 27-\mathrm{C} 32$ & $130.26(19)$ \\
\hline $\mathrm{C} 8-\mathrm{C} 1-\mathrm{C} 20-\mathrm{C} 21$ & $71.02(19)$ & $\mathrm{C} 39-\mathrm{C} 26-\mathrm{C} 27-\mathrm{C} 32$ & $-107.5(2)$ \\
\hline $\mathrm{C} 14-\mathrm{C} 1-\mathrm{C} 20-\mathrm{C} 21$ & $-167.89(14)$ & $\mathrm{C} 45-\mathrm{C} 26-\mathrm{C} 27-\mathrm{C} 32$ & $10.6(2)$ \\
\hline $\mathrm{C} 2-\mathrm{C} 1-\mathrm{C} 20-\mathrm{C} 22$ & $-175.46(14)$ & $\mathrm{C} 33-\mathrm{C} 26-\mathrm{C} 27-\mathrm{C} 28$ & $-52.2(2)$ \\
\hline $\mathrm{C} 8-\mathrm{C} 1-\mathrm{C} 20-\mathrm{C} 22$ & $-52.73(19)$ & $\mathrm{C} 39-\mathrm{C} 26-\mathrm{C} 27-\mathrm{C} 28$ & $70.04(19)$ \\
\hline $\mathrm{C} 14-\mathrm{C} 1-\mathrm{C} 20-\mathrm{C} 22$ & $68.36(18)$ & $\mathrm{C} 45-\mathrm{C} 26-\mathrm{C} 27-\mathrm{C} 28$ & $-171.86(15)$ \\
\hline $\mathrm{C} 8-\mathrm{C} 1-\mathrm{C} 2-\mathrm{C} 3$ & $-156.43(17)$ & $\mathrm{C} 33-\mathrm{C} 26-\mathrm{C} 45-\mathrm{C} 47$ & $69.82(19)$ \\
\hline $\mathrm{C} 14-\mathrm{C} 1-\mathrm{C} 2-\mathrm{C} 3$ & $83.5(2)$ & $\mathrm{C} 39-\mathrm{C} 26-\mathrm{C} 45-\mathrm{C} 47$ & $-53.8(2)$ \\
\hline $\mathrm{C} 20-\mathrm{C} 1-\mathrm{C} 2-\mathrm{C} 3$ & $-36.7(2)$ & $\mathrm{C} 27-\mathrm{C} 26-\mathrm{C} 45-\mathrm{C} 47$ & $-168.60(15)$ \\
\hline $\mathrm{C} 8-\mathrm{C} 1-\mathrm{C} 2-\mathrm{C} 7$ & $28.6(2)$ & $\mathrm{C} 33-\mathrm{C} 26-\mathrm{C} 45-\mathrm{C} 46$ & $-52.4(2)$ \\
\hline $\mathrm{C} 14-\mathrm{C} 1-\mathrm{C} 2-\mathrm{C} 7$ & $-91.5(2)$ & $\mathrm{C} 39-\mathrm{C} 26-\mathrm{C} 45-\mathrm{C} 46$ & $-176.05(16)$ \\
\hline $\mathrm{C} 20-\mathrm{C} 1-\mathrm{C} 2-\mathrm{C} 7$ & $148.34(17)$ & $\mathrm{C} 27-\mathrm{C} 26-\mathrm{C} 45-\mathrm{C} 46$ & $69.19(19)$ \\
\hline $\mathrm{C} 2-\mathrm{C} 1-\mathrm{C} 14-\mathrm{C} 19$ & $-108.4(2)$ & $\mathrm{C} 44-\mathrm{C} 39-\mathrm{C} 40-\mathrm{C} 41$ & $-3.1(3)$ \\
\hline $\mathrm{C} 8-\mathrm{C} 1-\mathrm{C} 14-\mathrm{C} 19$ & $130.6(2)$ & $\mathrm{C} 26-\mathrm{C} 39-\mathrm{C} 40-\mathrm{C} 41$ & $-177.85(17)$ \\
\hline $\mathrm{C} 20-\mathrm{C} 1-\mathrm{C} 14-\mathrm{C} 19$ & $11.6(2)$ & $\mathrm{C} 39-\mathrm{C} 26-\mathrm{C} 33-\mathrm{C} 38$ & $45.1(2)$ \\
\hline $\mathrm{C} 2-\mathrm{C} 1-\mathrm{C} 14-\mathrm{C} 15$ & $68.2(2)$ & $\mathrm{C} 27-\mathrm{C} 26-\mathrm{C} 33-\mathrm{C} 38$ & $161.54(16)$ \\
\hline $\mathrm{C} 8-\mathrm{C} 1-\mathrm{C} 14-\mathrm{C} 15$ & $-52.8(2)$ & $\mathrm{C} 45-\mathrm{C} 26-\mathrm{C} 33-\mathrm{C} 38$ & $-76.1(2)$ \\
\hline
\end{tabular}


$\mathrm{C} 20-\mathrm{C} 1-\mathrm{C} 14-\mathrm{C} 15$

$\mathrm{C} 2-\mathrm{C} 1-\mathrm{C} 8-\mathrm{C} 13$

$\mathrm{C} 14-\mathrm{C} 1-\mathrm{C} 8-\mathrm{C} 13$

$\mathrm{C} 20-\mathrm{C} 1-\mathrm{C} 8-\mathrm{C} 13$

$\mathrm{C} 2-\mathrm{C} 1-\mathrm{C} 8-\mathrm{C} 9$

$\mathrm{C} 14-\mathrm{C} 1-\mathrm{C} 8-\mathrm{C} 9$

$\mathrm{C} 20-\mathrm{C} 1-\mathrm{C} 8-\mathrm{C} 9$

$\mathrm{C} 23-\mathrm{O} 1-\mathrm{C} 22-\mathrm{C} 20$

$\mathrm{C} 21-\mathrm{C} 20-\mathrm{C} 22-\mathrm{O} 1$

$\mathrm{C} 1-\mathrm{C} 20-\mathrm{C} 22-\mathrm{O} 1$

$\mathrm{C} 13-\mathrm{C} 8-\mathrm{C} 9-\mathrm{C} 10$

$\mathrm{C} 1-\mathrm{C} 8-\mathrm{C} 9-\mathrm{C} 10$

$\mathrm{C} 23-\mathrm{O} 2-\mathrm{C} 21-\mathrm{C} 20$

$\mathrm{C} 22-\mathrm{C} 20-\mathrm{C} 21-\mathrm{O} 2$

$\mathrm{C} 1-\mathrm{C} 20-\mathrm{C} 21-\mathrm{O} 2$

$\mathrm{C} 7-\mathrm{C} 2-\mathrm{C} 3-\mathrm{C} 4$

$\mathrm{C} 1-\mathrm{C} 2-\mathrm{C} 3-\mathrm{C} 4$

$\mathrm{C} 9-\mathrm{C} 8-\mathrm{C} 13-\mathrm{C} 12$

$\mathrm{C} 1-\mathrm{C} 8-\mathrm{C} 13-\mathrm{C} 12$

C19-C14-C15-C16

$\mathrm{C} 1-\mathrm{C} 14-\mathrm{C} 15-\mathrm{C} 16$

$\mathrm{C} 3-\mathrm{C} 2-\mathrm{C} 7-\mathrm{C} 6$

$\mathrm{C} 1-\mathrm{C} 2-\mathrm{C} 7-\mathrm{C} 6$

$\mathrm{C} 4-\mathrm{C} 5-\mathrm{C} 6-\mathrm{C} 7$

$\mathrm{C} 2-\mathrm{C} 7-\mathrm{C} 6-\mathrm{C} 5$

$\mathrm{C} 12-\mathrm{C} 11-\mathrm{C} 10-\mathrm{C} 9$

$\mathrm{C} 8-\mathrm{C} 9-\mathrm{C} 10-\mathrm{C} 11$

$\mathrm{C} 6-\mathrm{C} 5-\mathrm{C} 4-\mathrm{C} 3$

$\mathrm{C} 2-\mathrm{C} 3-\mathrm{C} 4-\mathrm{C} 5$

$\mathrm{C} 10-\mathrm{C} 11-\mathrm{C} 12-\mathrm{C} 13$

$\mathrm{C} 8-\mathrm{C} 13-\mathrm{C} 12-\mathrm{C} 11$

$\mathrm{C} 14-\mathrm{C} 15-\mathrm{C} 16-\mathrm{C} 17$

C15-C14-C19-C18

$\mathrm{C} 1-\mathrm{C} 14-\mathrm{C} 19-\mathrm{C} 18$

$\mathrm{C} 14-\mathrm{C} 19-\mathrm{C} 18-\mathrm{C} 17$

$\mathrm{C} 19-\mathrm{C} 18-\mathrm{C} 17-\mathrm{C} 16$

$\mathrm{C} 15-\mathrm{C} 16-\mathrm{C} 17-\mathrm{C} 18$

$\mathrm{C} 33-\mathrm{C} 26-\mathrm{C} 39-\mathrm{C} 44$

$\mathrm{C} 27-\mathrm{C} 26-\mathrm{C} 39-\mathrm{C} 44$

$\mathrm{C} 45-\mathrm{C} 26-\mathrm{C} 39-\mathrm{C} 44$

$\mathrm{C} 33-\mathrm{C} 26-\mathrm{C} 39-\mathrm{C} 40$

$\mathrm{C} 27-\mathrm{C} 26-\mathrm{C} 39-\mathrm{C} 40$

$\mathrm{C} 45-\mathrm{C} 26-\mathrm{C} 39-\mathrm{C} 40$
$-171.80(16)$

$-141.65(18)$

$-25.1(2)$

96.32 (19)

$44.2(2)$

$160.70(16)$

$-77.8(2)$

$-58.7(2)$

54.90 (19)

$-176.66(14)$

-0.4 (3)

$173.99(17)$

$57.8(2)$

$-54.6(2)$

$177.79(14)$

$-2.3(3)$

$-177.53(18)$

0.1 (3)

$-174.26(18)$

$-3.2(3)$

$180.00(18)$

$1.9(3)$

177.14 (18)

$-0.9(3)$

$-0.3(3)$

$0.2(4)$

$0.3(3)$

$0.5(3)$

$1.1(3)$

$-0.5(4)$

$0.4(3)$

$0.9(3)$

$3.5(3)$

$-179.8(2)$

$-1.7(4)$

-0.8 (4)

$1.2(4)$

$18.5(2)$

$-101.8(2)$

$138.43(18)$

$-167.18(16)$

$72.56(19)$

$-47.2(2)$
$\mathrm{C} 39-\mathrm{C} 26-\mathrm{C} 33-\mathrm{C} 34$

$\mathrm{C} 27-\mathrm{C} 26-\mathrm{C} 33-\mathrm{C} 34$

$\mathrm{C} 45-\mathrm{C} 26-\mathrm{C} 33-\mathrm{C} 34$

$\mathrm{C} 48-\mathrm{O} 3-\mathrm{C} 47-\mathrm{C} 45$

$\mathrm{C} 46-\mathrm{C} 45-\mathrm{C} 47-\mathrm{O} 3$

$\mathrm{C} 26-\mathrm{C} 45-\mathrm{C} 47-\mathrm{O} 3$

$\mathrm{C} 48-\mathrm{O} 4-\mathrm{C} 46-\mathrm{C} 45$

$\mathrm{C} 47-\mathrm{C} 45-\mathrm{C} 46-\mathrm{O} 4$

$\mathrm{C} 26-\mathrm{C} 45-\mathrm{C} 46-\mathrm{O} 4$

$\mathrm{C} 30-\mathrm{C} 29-\mathrm{C} 28-\mathrm{C} 27$

$\mathrm{C} 32-\mathrm{C} 27-\mathrm{C} 28-\mathrm{C} 29$

$\mathrm{C} 26-\mathrm{C} 27-\mathrm{C} 28-\mathrm{C} 29$

$\mathrm{C} 34-\mathrm{C} 33-\mathrm{C} 38-\mathrm{C} 37$

$\mathrm{C} 26-\mathrm{C} 33-\mathrm{C} 38-\mathrm{C} 37$

$\mathrm{C} 40-\mathrm{C} 39-\mathrm{C} 44-\mathrm{C} 43$

$\mathrm{C} 26-\mathrm{C} 39-\mathrm{C} 44-\mathrm{C} 43$

$\mathrm{C} 39-\mathrm{C} 40-\mathrm{C} 41-\mathrm{C} 42$

$\mathrm{C} 39-\mathrm{C} 44-\mathrm{C} 43-\mathrm{C} 42$

$\mathrm{C} 44-\mathrm{C} 43-\mathrm{C} 42-\mathrm{C} 41$

$\mathrm{C} 40-\mathrm{C} 41-\mathrm{C} 42-\mathrm{C} 43$

$\mathrm{C} 28-\mathrm{C} 27-\mathrm{C} 32-\mathrm{C} 31$

$\mathrm{C} 26-\mathrm{C} 27-\mathrm{C} 32-\mathrm{C} 31$

$\mathrm{C} 38-\mathrm{C} 33-\mathrm{C} 34-\mathrm{C} 35$

$\mathrm{C} 26-\mathrm{C} 33-\mathrm{C} 34-\mathrm{C} 35$

$\mathrm{C} 27-\mathrm{C} 32-\mathrm{C} 31-\mathrm{C} 30$

$\mathrm{C} 28-\mathrm{C} 29-\mathrm{C} 30-\mathrm{C} 31$

$\mathrm{C} 32-\mathrm{C} 31-\mathrm{C} 30-\mathrm{C} 29$

$\mathrm{C} 33-\mathrm{C} 38-\mathrm{C} 37-\mathrm{C} 36$

$\mathrm{C} 35-\mathrm{C} 36-\mathrm{C} 37-\mathrm{C} 38$

$\mathrm{C} 37-\mathrm{C} 36-\mathrm{C} 35-\mathrm{C} 34$

$\mathrm{C} 33-\mathrm{C} 34-\mathrm{C} 35-\mathrm{C} 36$

$\mathrm{C} 46-\mathrm{O} 4-\mathrm{C} 48-\mathrm{O} 3$

$\mathrm{C} 46-\mathrm{O} 4-\mathrm{C} 48-\mathrm{C} 50$

$\mathrm{C} 46-\mathrm{O} 4-\mathrm{C} 48-\mathrm{C} 49$

$\mathrm{C} 47-\mathrm{O} 3-\mathrm{C} 48-\mathrm{O} 4$

$\mathrm{C} 47-\mathrm{O} 3-\mathrm{C} 48-\mathrm{C} 50$

$\mathrm{C} 47-\mathrm{O} 3-\mathrm{C} 48-\mathrm{C} 49$

$\mathrm{C} 22-\mathrm{O} 1-\mathrm{C} 23-\mathrm{O} 2$

$\mathrm{C} 22-\mathrm{O} 1-\mathrm{C} 23-\mathrm{C} 25$

$\mathrm{C} 22-\mathrm{O} 1-\mathrm{C} 23-\mathrm{C} 24$

$\mathrm{C} 21-\mathrm{O} 2-\mathrm{C} 23-\mathrm{O} 1$

$\mathrm{C} 21-\mathrm{O} 2-\mathrm{C} 23-\mathrm{C} 25$

$\mathrm{C} 21-\mathrm{O} 2-\mathrm{C} 23-\mathrm{C} 24$
$-141.63(17)$

$-25.2(2)$

$97.21(19)$

$57.8(2)$

$-54.4(2)$

$179.86(14)$

$-59.9(2)$

$55.2(2)$

$-178.10(15)$

0.7 (3)

$-2.2(3)$

-179.92 (17)

$0.2(3)$

$173.79(18)$

2.9 (3)

177.44 (19)

0.5 (3)

-0.2 (4)

$-2.5(3)$

2.3 (3)

$2.2(3)$

179.79 (18)

-0.8 (3)

$-174.35(18)$

-0.7 (3)

0.8 (3)

$-0.8(4)$

$0.6(3)$

$-0.9(4)$

$0.4(4)$

$0.5(3)$

$58.7(2)$

172.54 (18)

$-65.6(2)$

$-57.6(2)$

$-171.43(17)$

66.8 (2)

$56.8(2)$

170.43 (16)

$-67.0(2)$

$-56.3(2)$

$-170.34(17)$

68.1 (2) 